\title{
Tamiz de los errores innatos del metabolismo por espectrometría de masas en tándem: principales biomarcadores
}

'Departamento de Genética Bioquímica. Centro Nacional de Genética Médica. Campus del ICBP Victoria de Girón. La Habana, Cuba. aLicenciado en Bioquímica. Máster en Bioquímica Clínica, Facultad de Biología, Universidad de La Habana, Cuba.

Recibido el 15 de marzo de 2011. Aceptado el 1 de junio de 2011

Correspondencia a: Derbis Campos Hernández, Centro Nacional de Genética Médica. Campus del ICBP Victoria de Girón. Calle 146 No. 3102, Playa. La Habana 16, C.P: 1600 Cuba. Teléfono: 2089991 E-mail: derbisch@cngen. sld.cu

\author{
DERBIS CAMPOS H. ${ }^{1, \mathrm{a}}$
}

The use of tandem mass spectrometry for the diagnosis of inborn errors of metabolism has the potential to expand the newborn screening panel to include a vast number of diseases. This technology allows the detection, in the same spot of dried blood on filter paper and during one single analytical run, of different metabolic diseases. Tandem mass spectrometry is rapidly replacing the classical screening techniques approach of one-metabolite detected per analysis per disease by its ability of simultaneous quantification of several metabolites as markers of many diseases, such as acylcarnitines and amino acids. It is clear that a single metabolite can be a biomarker for several diseases, so the multiplex approach of using tandem mass spectrometry enhances, on average, the sensitivity and specificity of the screening. However, there are differences for particular metabolites and the diseases they detect within the same method. Disorders such as the tyrosinemias and among the organic acidemias, the methylmalonic acidemias, have a substantially higher false-positive rate than other more common metabolic diseases such as medium-chain acyl-CoA dehydrogenase deficiency and phenylketonuria. Before introducing this technology into routine newborn screening programs it is necessary to consider the frequency of each disease, as well as the response to early treatment or variables related to the collection of the sample.

(Rev Med Chile 2011; 139: 1356-1364).

Key words: Amino acid metabolism, inborn errors; Phenylketonurias; Spectrum analysis.

$\Lambda$ ctualmente, existen varios países en los que se ha implementado o se encuentra en desarrollo la incorporación de la espectrometría de masas en tándem (MS/MS) en los programas de tamiz neonatal ${ }^{1}$.

Las técnicas de tamiz clásicas tradicionalmente se han basado en realizar un ensayo por cada muestra para detectar un único metabolito marcador de una sola enfermedad. La MS/MS ofrece la potencialidad de reemplazar este enfoque mediante la realización de un ensayo para detectar varios biomarcadores por cada enfermedad y poder diagnosticar más de 40 enfermedades simultánea- mente, en la misma muestra de sangre seca sobre papel de filtro ${ }^{2}$. Esto facilita la incorporación de nuevas enfermedades a los paneles de tamiz neonatal existentes.

El concepto de "un ensayo para múltiples enfermedades" que se materializa a través del perfil metabólico obtenido por MS/MS ha amplificado la responsabilidad de los programas de tamiz neonatal en relación a lograr la máxima sensibilidad y especificidad para la identificación de los pacientes minimizando al mismo tiempo la tasa de falsos positivo ${ }^{2}$. La obtención de falsos positivo produce un estado de ansiedad en la familia así como un 
gasto adicional en términos de tiempo y esfuerzo profesional para la obtención de nuevas muestras para su reanálisis y seguimiento. La obtención de falsos negativo es aun más costosa ya que involucra la no detección a tiempo de pacientes con errores innatos del metabolismo (EIM) potencialmente tratables ${ }^{3}$.

\section{Biomarcadores de aminoacidemias}

El biomarcador principal en las aminoacidemias es aquel aminoácido que se acumula producto del defecto metabólico. Sin embargo, si se considera el perfil de otros aminoácidos o las razones entre ellos como biomarcadores secundarios se disminuye la aparición de falsos positivo (Tabla 1). Se ha demostrado que una concentración significativamente elevada de fenilalanina junto con una razón molar fenilalanina/tirosina elevada es un indicador más sensible para el diagnóstico de la fenilcetonuria (PKU) que la determinación únicamente de la fenilalanina ${ }^{4}$. Esto permite diferenciar pacientes con hiperfenilalaninemia de tipo no PKU de pacientes con PKU con una sensibilidad de $100 \%{ }^{4}$. Mediante esta metodología, Zytkovicz y cols lograron aumentar de 20 a $28 \%$ el valor predictivo positivo para la $\mathrm{PKU}^{5}$ mientras que Schulze y cols obtuvieron una especificidad de $99,95 \%$ y un valor predictivo positivo de 32,35 , uno de los más altos del estudio ${ }^{3}$.

Otras relaciones que frecuentemente se utilizan y que han mostrado ser útiles en aumentar el valor predictivo positivo en comparación con el análisis individual de cada aminoácido son: metionina/ fenilalanina y metionina/(leucina+isoleucina) para el diagnóstico de la hipermetioninemia y de la homocistinuria respectivamente; ornitina/ citrulina y citrulina/arginina para la citrulinemia, arginina/ornitina para la argininemia y (leucina+isoleucina)/fenilalanina para la enfermedad de la orina con olor a jarabe de arce ${ }^{5-8}$.

El análisis completo del perfil de aminoácidos también permite reconocer una elevación generalizada en la concentración de aminoácidos debido a causas iatrogénicas. Un situación muy común resulta la administración de una nutrición parenteral total que podría constituir una fuente de falsos positivo si se analizara un solo aminoácido en particular.

En el estudio realizado por Zytkovicz y cols, el
$34 \%$ de los neonatos detectados inicialmente por un incremento en el contenido de aminoácidos fueron considerados como hiperalimentados, al observarse un aumento en la concentración de dos o más especies diferentes 5 . Aunque la composición y dosis de la preparación farmacológica determinará si el perfil de aminoácidos muestra una elevación moderada o severa, las razones molares entre ellos permanecerá normal. Algunas de estas preparaciones son insuficientes en tirosina lo cual produce una relación fenilalanina/tirosina elevada. Sin embargo, al mantenerse normales otras razones como fenilalanina/leucina, se elimina la posibilidad de un caso de $\mathrm{PKU}^{5}$.

Los neonatos que reciben nutrición parenteral total frecuentemente presentan concentraciones elevadas de los aminoácidos leucina, isoleucina y valina ${ }^{9}$. El incremento de estos aminoácidos se utiliza como criterio para el diagnóstico por MS/ MS de la enfermedad de la orina con olor a jarabe de arce. Por lo tanto, esta variable debe tomarse en cuenta para disminuir la tasa de falsos positivos. El único marcador completamente específico para esta enfermedad es la alo-isoleucina, aminoácido isobárico con los anteriores y que no puede ser cuantificado de forma independiente por los métodos tradicionales de tamiz por MS/MS ${ }^{10}$.

Un aumento en la concentración de tirosina puede indicar indistintamente la presencia de tirosinemias de tipo I, II o III. Por lo tanto, la detección por MS/MS de este aminoácido no permite discriminar al tipo I, de mayor prevalencia, del resto ${ }^{11}$. Además, la elevación de forma significativa de la concentración de tirosina en muchos de estos pacientes se produce de forma lenta en el tiempo, lo cual ha inducido a la obtención de falsos negativos en laboratorios que analizan muestras tomadas hasta el segundo día de nacido ${ }^{12}$. También se ha reportado una alta tasa de resultados falsos positivos producto de la alta incidencia en la población neonatal de tirosinemia transitoria, un trastorno metabólico benigno ${ }^{13}$. Esto hace que el tamiz por métodos tradicionales de MS/MS para la tirosinemia tipo I sea bastante discutido, aún cuando su detección temprana posibilita una intervención terapéutica muy efectiva en los pacientes.

El único metabolito completamente específico para la detección de tirosinemia tipo I es la succinilcetona. A partir del método desarrollado por Allard y cols, para la determinación cuantitativa por MS/MS de este metabolito, de forma 
Tabla 1. Errores innatos del metabolismo disponibles para su tamiz neonatal mediante el análisis del perfil de aminoácidos y acilcarnitinas por MS/MS

\begin{tabular}{|c|c|c|c|}
\hline EIM & Deficiencia metabólica & $\begin{array}{l}\text { Biomarcadores } \\
\text { primarios }\end{array}$ & $\begin{array}{l}\text { Biomarcadores } \\
\text { secundarios }\end{array}$ \\
\hline \multicolumn{4}{|c|}{ Degradación de aminoácidos/ciclo de la urea } \\
\hline Argininemia (ARG) & Arginasa & Arg & Arg/Orn \\
\hline Aciduria arginosuccinica (ASA) & Arginosuccinato liasa & ASA & \\
\hline $\begin{array}{l}\text { Citrulinemia tipo I (CIT-I) / Citruli- } \\
\text { nemia tipo II (CIT-II) }\end{array}$ & Arginosuccinato sintetasa & Cit & Orn/Cit, Cit/Arg \\
\hline $\begin{array}{l}\text { Enfermedad de orina con olor a } \\
\text { jarabe de arce (MSUD) }\end{array}$ & $\begin{array}{l}\text { Deshidrogenasa de ceto-ácidos de } \\
\text { cadena ramificada }\end{array}$ & (Leu, Ile, allo-Ile)a , Val & (Leu, Ile, allo-lle)/Phe \\
\hline Homocistinuria (HCY) & $\begin{array}{l}\text { Sistationina } \\
\text { b-sintasa }\end{array}$ & Met & $\begin{array}{l}\text { Met/Phe, } \\
\text { Met/(Leu, Ile, allo-Ile) }\end{array}$ \\
\hline Hipermetioninemia (MET) & Metionina adenosil transferasa & & \\
\hline $\begin{array}{l}\text { Fenilcetonuria (PKU) /Hiperfenila- } \\
\text { laninemia (HPA) }\end{array}$ & $\begin{array}{l}\text { Fenilalanina hidroxilasa (> 98\%), } \\
\text { defectos en } \mathrm{BH} 4\end{array}$ & Phe, Phe/Tyr & \\
\hline Tirosinemia tipo I (TYR-I) & Fumarilacetoacetato hidrolasa & SUAC & Tyr \\
\hline Tirosinemia tipo II (TYR-II) & Tirosina aminotransferasa & Tyr & \\
\hline Tirosinemia tipo III (TYR-III) & $\begin{array}{l}\text { Ácido 4-hidroxifenilpirúvico } \\
\text { oxidasa }\end{array}$ & & \\
\hline \multicolumn{4}{|c|}{ Degradación de ácidos orgánicos } \\
\hline Acidemia malónica (MA) & Malonil-CoA descarboxilasa & C3-DC & \\
\hline Acidemia propiónica (PA) & Propionil-CoA carboxilasa & $\mathrm{C} 3, \mathrm{C} 3 / \mathrm{C} 2$ & $\mathrm{C} 3 / \mathrm{CO}, \mathrm{C} 3 / \mathrm{C} 16$ \\
\hline Acidemia metilmalónica (MMA) & $\begin{array}{l}\text { Metilmalonil-CoA mutasa, síntesis } \\
\text { adenosilcobalamina y/o metilco- } \\
\text { balamina. }\end{array}$ & & \\
\hline Acidemia isovalérica (IVA) & Isovaleril-CoA deshidrogenasa & $C 5^{b}$ & $\mathrm{C} 5 / \mathrm{C} 2$ \\
\hline $\begin{array}{l}\text { Deficiencia de 2-metilbutiril-CoA } \\
\text { deshidrogenasa (MBD) }\end{array}$ & 2-metilbutiril-CoA deshidrogenasa & & \\
\hline $\begin{array}{l}\text { Deficiencia de 3-hidroxi-3-metil- } \\
\text { glutaril-CoA liasa (HMGL) }\end{array}$ & 3-hidroxi-3-metilglutaril-CoA liasa & $\mathrm{C} 5-\mathrm{OH}^{\mathrm{c}}$ & C5-3M-DC \\
\hline $\begin{array}{l}\text { Deficiencia de 3-metilcrotonil-CoA } \\
\text { carboxilasa (MCC) }\end{array}$ & 3-metilcrotonil-CoA carboxilasa & $\mathrm{C} 5-\mathrm{OH}$ & \\
\hline $\begin{array}{l}\text { Aciduria 3-metil glutacónica } \\
\text { (MGA) }\end{array}$ & 3-metilglutaconil-CoA hidratasa & & \\
\hline $\begin{array}{l}\text { Deficiencia de 2-metil-3-hidro- } \\
\text { xibutiril-CoA deshidrogenasa } \\
\text { (MHBD) }\end{array}$ & $\begin{array}{l}\text { 2-metil-3-hidroxibutiril-CoA deshi- } \\
\text { drogenasa }\end{array}$ & $\mathrm{C} 5: 1, \mathrm{C} 5-\mathrm{OH}$ & \\
\hline Deficiencia de b-ceto tiolasa (BKT) & $\begin{array}{l}\text { Acetoacetil-CoA tiolasa mitocon- } \\
\text { drial }\end{array}$ & & \\
\hline $\begin{array}{l}\text { Deficiencia múltiple de CoA car- } \\
\text { boxilasas (MCD) }\end{array}$ & Holocarboxilasa sintetasa & $\mathrm{C} 3, \mathrm{C} 5-\mathrm{OH}$ & $\mathrm{C} 3 / \mathrm{C} 2$ \\
\hline Acidemia glutárica tipo I (GA-I) & Glutaril-CoA deshidorgenasa & C5-DC & $C 5-D C / C 8, C 5-D C / C 16$ \\
\hline $\begin{array}{l}\text { Deficiencia de isobutiril-CoA } \\
\text { deshidrogenasa (IBD) }\end{array}$ & Isobutiril-CoA deshidrogenasa & C4 & $\mathrm{C} 4 / \mathrm{C} 3$ \\
\hline
\end{tabular}


Espectrometría de masas en tándem - D. Campos et al

Tabla 1. Errores innatos del metabolismo disponibles para su tamiz neonatal mediante el análisis del perfil de aminoácidos y acilcarnitinas por MS/MS (continuación)

\begin{tabular}{|c|c|c|c|}
\hline EIM & Deficiencia metabólica & $\begin{array}{l}\text { Biomarcadores } \\
\text { primarios }\end{array}$ & $\begin{array}{l}\text { Biomarcadores } \\
\text { secundarios }\end{array}$ \\
\hline \multicolumn{4}{|c|}{ Degradación mitocondrial de ácidos grasos } \\
\hline $\begin{array}{l}\text { Deficiencia de acil-CoA deshidro- } \\
\text { genasa de cadena corta (SCAD) }\end{array}$ & $\begin{array}{l}\text { Acil-CoA deshidrogenasa de } \\
\text { cadena corta }\end{array}$ & $\mathrm{C} 4$ & $\mathrm{C} 4 / \mathrm{C} 3$ \\
\hline $\begin{array}{l}\text { Deficiencia de acil-CoA deshidro- } \\
\text { genasa de cadena media (MCAD) }\end{array}$ & $\begin{array}{l}\text { Acil-CoA deshidrogenasa de } \\
\text { cadena media }\end{array}$ & $\mathrm{C} 8$ & $\begin{array}{l}\mathrm{C} 6, \mathrm{C} 10, \mathrm{C} 10: 1, \mathrm{C} 8 / \\
\mathrm{C} 10\end{array}$ \\
\hline $\begin{array}{l}\text { Deficiencia de acil-CoA deshi- } \\
\text { drogenasa de cadena muy larga } \\
\text { (VLCAD) }\end{array}$ & $\begin{array}{l}\text { Acil-CoA deshidrogenasa de } \\
\text { cadena muy larga }\end{array}$ & $\mathrm{C} 14: 1$ & $\begin{array}{l}\text { C14, C16, C12, C14:2, } \\
\text { C18, C14:1/C16, C18:1 }\end{array}$ \\
\hline $\begin{array}{l}\text { Deficiencia múltiple de acil-CoA } \\
\text { deshidrogenasas (MADD) o acide- } \\
\text { mia glutárica tipo II (GA-II) }\end{array}$ & $\begin{array}{l}\text { Flavoproteína transferidora de } \\
\text { electrones (ETF), ETF deshidro- } \\
\text { genasa. }\end{array}$ & $\mathrm{C} 8, \mathrm{C} 10, \mathrm{C} 12$ & $\begin{array}{l}C 4, C 5, C 5-D C, C 6 \\
C 10, C 14, C 16\end{array}$ \\
\hline $\begin{array}{l}\text { Deficiencia de carnitina palmitoil } \\
\text { transferasa II (CPT-II) }\end{array}$ & Carnitina palmitoil transferasa 2 & C16 & $\mathrm{C} 18, \mathrm{C} 18: 1$ \\
\hline $\begin{array}{l}\text { Deficiencia de translocasa de } \\
\text { carnitina/acilcarnitina (CATD) }\end{array}$ & $\begin{array}{l}\text { Translocasa de carnitina/ } \\
\text { acilcarnitina }\end{array}$ & & \\
\hline $\begin{array}{l}\text { Deficiencia de carnitina palmitoil } \\
\text { transferasa I (CPT-I) }\end{array}$ & Carnitina palmitoil transferasa 1 & $\mathrm{CO} /(\mathrm{C} 16+\mathrm{C} 18)$ & \\
\hline $\begin{array}{l}\text { Deficiencia del transportador plas- } \\
\text { mático de carnitina (CTD) }\end{array}$ & $\begin{array}{l}\text { Transportador de carnitina depen- } \\
\text { diente de iones sodio (OCTN2) }\end{array}$ & $\mathrm{C} 0^{d}, \mathrm{C}^{1} 6^{d}, C 18^{d}$ & \\
\hline $\begin{array}{l}\text { Deficiencia de proteína mitocon- } \\
\text { drial trifuncional o de alguna de } \\
\text { sus actividades (MTPD) }\end{array}$ & $\begin{array}{l}\text { Proteína mitocondrial trifuncional } \\
\text { con actividades: 3-hidroxi-acil- } \\
\text { CoA deshidrogenasa, enoil-CoA } \\
\text { hidratasa y 3-oxo-acil-CoA tiolasa } \\
\text { de cadena larga }\end{array}$ & $\begin{array}{l}\mathrm{C} 16-\mathrm{OH} \\
\mathrm{C} 16: 1-\mathrm{OH} \\
\mathrm{C} 18-\mathrm{OH} \\
\mathrm{C} 18: 1-\mathrm{OH}\end{array}$ & \\
\hline
\end{tabular}

Arg: arginina, Orn: ornitina, ASA: ácido arginosuccínico, Cit: citrulina, Met: metionina, Phe: fenilalanina, Leu: leucina, lle: isoleucina, alo-lle: alo-isoleucina, Val: valina, Tyr: tirosina, SUAC: succinilacetona, CO: carnitina, C2: acetilcarnitina, C3: propionilcarnitina, C3-DC: malonilcarnitina, C4: butirilcarnitina, C5: isovalerilcarnitina/3-metilbutirilcarnitina, C5-DC: glutarilcarnitina, C5:1: tiglicarnitina, C5-OH: 3-hidroxi-isovalerilcarnitina/2-metil-3-hidroxi-butirilcarnitina, C5-3M-DC: 3-metil-glutarilcarnitina, C6: hexanoilcarnitina, C8: octanoilcarnitina, C10: decanoilcarnitina, C10:1: decenoilcarnitina, C12: dodecanoilcarnitina, C14: tetradecanoilcarnitina, C14:1: tetradecenoilcarnitina, C14:2: tetradodecenoilcarnitina, C16: hexadecanoilcarnitina, C16-OH: 3-hidroxi-hexadecanoilcarnitina, C18: octadecanoilcarnitina: C18:1: octadecenoilcarnitina, C18-OH: 3-hidroxi-octadecanoilcarnitina, C18:1-OH: 3-hidroxi-octadecenoilcarnitina. alos aminoácidos Leu, lle y allo-Ile son isobáricos e indistinguibles por MS/ MS. bla isovalerilcarnitina que se acumula en la IVA es isobárica con la 3-metilbutirilcarnitina que se acumula en la MBD. cla 3-hidroxi-isovalerilcarnitina que se acumula en las HMGC, MCC y MGA es isobárica con la 2-metil-3-hidroxi-butirilcarnitina que se acumula en las MHBD y BKT. den la CTD lo que se detecta es una disminución de estos metabolitos.

independiente en sangre seca sobre papel de filtro $^{14}$; se han introducido y validado importantes modificaciones que posibilitan la determinación simultánea de succinilcetona junto con el resto de los aminoácidos y acilcarnitinas ${ }^{15,16}$. La utilización de este marcador en el tamiz neonatal de la tirosinemia tipo I disminuye notablemente la ocurrencia de falsos negativo y falsos positivo ${ }^{17}$.

\section{Biomarcadores de acidemias orgánicas y defectos en la oxidación de los ácidos grasos}

En las acidemias orgánicas, y especialmente en las deficiencias en la oxidación de los ácidos grasos, aunque existe una especie de acilcarnitina clave sobre la cual basar el diagnóstico, ocurre el incremento de otras especies. Sin embargo, la 
variación de algunos de estos metabolitos no es específica para una única enfermedad. Por tal motivo, cobra mayor importancia analizar el perfil completo de acilcarnitinas y las diferentes razones molares de interés diagnóstico para el tamiz de la enfermedad (Tabla 1).

Los principales metabolitos relacionados con el diagnóstico de la deficiencia de acil-CoA deshidrogenasa de cadena media (MCAD) son las especies hexanoilcarnitina (C6), octanoilcarnitina (C8), decanoilcarnitina (C10) y decenoilcarnitina $(\mathrm{C} 10: 1)^{18}$. Tratamientos farmacológicos como la administración de ácido valproico, aceite de triglicéridos de cadena media y carnitina también producen un incremento de $\mathrm{C} 8, \mathrm{C} 6$ y $\mathrm{C} 10$ interfiriendo con el diagnóstico de la $\mathrm{MCAD}^{19}$. Sin embargo, la relación C8/C10 se incrementa significativamente en los pacientes con MCAD a diferencia de aquellos que reciben estos medicamentos $^{20}$. Esta relación también permite distinguir a la MCAD de otras deficiencias metabólicas que producen un incremento en $\mathrm{C} 8$ como la deficiencia múltiple de acil-CoA deshidrogenasas (MADD). Su utilización junto con la relación C8/ acetilcarnitina (C2) también ha permitido discriminar entre falsos y verdaderos casos positivos e identificar dentro de estos las subpoblaciones de MCAD leve y clásica ${ }^{21}$.

La butirilcarnitina (C4) determinada por MS/ MS permite identificar las deficiencias de acil-CoA deshidrogenasa de cadena corta (SCAD) y de isobutiril-CoA deshidrogenasa ${ }^{22}$. Este marcador también puede incrementarse en otras enfermedades asociado a otras especies de acilcarnitinas, por lo que el perfil de MS/MS obtenido puede ayudar a su discriminación y sugerir cual enfermedad es la más probable. Por ejemplo, un aumento de las acilcarnitinas de cadena media y larga junto a $\mathrm{C} 4$ puede reflejar una $\mathrm{MADD}^{23}$; mientras que durante la administración de L-carnitina se incrementan además de C4 las especies propionilcarnitina (C3) y $\mathrm{C}_{2}{ }^{19}$. Un elemento útil para diferenciar una SCAD verdadera y reducir los resultados falsos positivos es expresar la razón de C4 ante otras acilcarnitinas de cadena corta ${ }^{19}$.

En la deficiencia de carnitina palmitoiltransferasa I (CPT-I) se produce una disminución en la concentración de las acilcarnitina de cadena larga, especialmente de hexadecanoilcarnitina (C16) y octadecanoilcarnitina (C18), junto a un significativo incremento de carnitina libre (C0) y tota ${ }^{24}$. Sin embargo, la cuantificación de $\mathrm{C} 0$ en muestras de sangre seca sobre papel de filtro no es confiable debido a la hidrólisis espontánea de determinadas acilcarnitinas durante la conservación de la muestra o su derivatización ${ }^{25}$. En un estudio publicado por Fingerhut y cols, la utilización de la relación $\mathrm{C} 0 /(\mathrm{C} 16+\mathrm{C} 18)$ permitió incrementar la especificidad y el valor predictivo positivo ${ }^{26}$. De esta forma se eliminó totalmente la ocurrencia de falsos positivo en comparación a un diagnóstico basado solamente en la disminución de la concentración de ambas especies de acilcarnitinas de cadena larga. Esta razón también permitió discriminar entre pacientes de CPT-I e individuos bajo tratamiento con suplemento de L-carnitina ${ }^{26}$. Un elemento esencial para asegurar la fiabilidad de esta relación es contar con un sistema de MS/ MS optimizado para determinar con exactitud las bajas concentraciones de estos metabolitos.

Las acilcarnitinas C16 y C18 también son los principales marcadores para el diagnóstico de la deficiencia de carnitina palmitoiltransferasa II y la deficiencia de carnitina-acilcarnitina traslocasa; para lo cual también se utiliza la octadecenoilcarnitina $(\mathrm{C} 18: 1)^{27}$. A diferencia de la CPT-1, en estas dos deficiencias se produce un aumento significativo de la concentración de las acilcarnitinas de cadena larga que permite su diferenciación. Sin embargo, la MS/MS no permite discriminar entre ambas por lo que se requiere un diagnóstico diferencial posterior por otro método ${ }^{28}$.

En este caso también se encuentra la acidemia propiónica y el grupo de las acidemias metilmalónicas, que incluye a las deficiencias de las enzimas metilmalonil-CoA mutasa, holocarboxilasa y biotinidasa; así como defectos en la síntesis de adenosilcobalamina y la deficiencia de vitamina $\mathrm{B}_{12}$. En todas estas enfermedades el marcador principal en el tamiz neonatal por MS/MS es el C $3^{29,30}$.

Precisamente, el balance desfavorable entre la especificidad y la sensibilidad de C3 como biomarcador primario ha sido esgrimido como una razón importante para excluir este grupo de enfermedades de algunos programas de tamiz. En el 2004, la tasa de reanálisis de 11 laboratorios en Alemania para un resultado de C3 elevado osciló entre 0,04 y 0,18 en comparación con una variación de 0,04 a 0,69 para el resto de los biomarcadores usados en el tamiz por MS/MS tomados en conjunto ${ }^{31}$. Un elevado punto de corte para este metabolito puede resultar en una significativa tasa de falsos 
negativos para las enfermedades del metabolismo de la cobalamina y para las formas menos severas o de presentación tardía de las acidurias propiónicas y metilmalónicas. Un punto de corte muy bajo provocaría una alta tasa de resultados de pesquisa de falsos positivos ${ }^{32}$.

La utilización de las razones de C3 frente a otras acilcarnitinas $(\mathrm{C} 3 / \mathrm{C} 2, \mathrm{C} 3 / \mathrm{C} 0$ o $\mathrm{C} 3 / \mathrm{C} 16)$ como marcadores secundarios permite mejorar la especificidad de este diagnóstico ${ }^{3,6,13,33}$. En el programa de tamiz neonatal australiano se sustituyó completamente a C3 por la razón C3/C2 como marcador primario para mejorar la sensibilidad ${ }^{34}$.

Mediante procedimientos estadísticos de minería de datos se han identificado nuevas combinaciones de marcadores que permiten incrementar la especificidad para un $100 \%$ de sensibilidad. En este sentido, Lindner y cols ratificaron una ganancia en especificidad al emplear la razón C3/C2 en lugar de únicamente C3 $(99,76 \%$ vs $98,47 \%)^{32}$. Nuevas combinaciones múltiples como [C3/C0, C3/C2 y 3-hidroxi-hexadecenoilcarnitina $(\mathrm{C} 16: 1 \mathrm{OH}) / \mathrm{C} 2]$ y $[\mathrm{C} 3 / \mathrm{C} 2, \mathrm{C} 3 / \mathrm{C} 0$ y $\mathrm{C} 16: 1 \mathrm{OH} /$ metilmalonilcarnitina (C4DC)] permitieron elevar la especificidad a $99,99 \%{ }^{32}$. No obstante, estas nuevas combinaciones determinadas por derivaciones aritméticas necesitan ser validadas en un mayor número de muestras positivas, así como encontrar una explicación bioquímica para la variación de especies como C16:1OH y C4DC. No obstante, el uso de herramientas bioinformáticas más complejas, que incluyen el desarrollo de nuevos algoritmos especializados y su aplicación a una creciente base de datos de tamiz por MS/ MS, constituye un acercamiento importante a la obtención de nuevos biomarcadores para ésta y otras enfermedades heredo-metabólicas ${ }^{35}$.

La concentración media de C3 en muestras de pacientes con aciduria propiónica es generalmente mayor que cuando existe una aciduria de tipo metilmalónica. Sin embargo, la variación en este marcador y en las relaciones de acilcarnitinas asociadas es lo suficientemente amplia como para imposibilitar una distinción confiable entre ambas $^{36}$. La metilmalonilcarnitina tampoco ha sido útil como marcador discriminativo, pues no se ha podido comprobar que su concentración aumente producto de un defecto del metabolismo del ácido metilmalónico ${ }^{36}$.

En relación a la isovalerilcarnitina (C5), su incremento en el perfil de acilcarnitinas puede ser indicativo de una acidemia isovalerica o de una deficiencia de 2-metilbutiril-CoA deshidrogenasa pues $\mathrm{C} 5$ y la butirilcarnitina son isómeros indistinguibles dentro de un perfil tradicional de tamiz por MS/MS ${ }^{37,38}$. La pivaloilcarnitina, derivado del ácido piválico presente en algunos antibióticos administrados al neonato o durante la gestación, también produce una señal isobárica. En Japón, el uso de este tipo de antibióticos en los neonatos durante un programa piloto de tamiz por MS/MS produjo una elevada tasa de falsos positivo para la acidemia isovalérica: 0,2 al $1 \%{ }^{39}$.

\section{Otros aspectos relacionados con el análisis de biomarcadores}

El análisis inicial por MS/MS es sólo la primera etapa para llegar a un diagnóstico positivo definitivo. En aquellos individuos cuyos resultados se encuentran por encima del nivel de corte establecido se solicita una nueva muestra para su reanálisis, generalmente mediante otra técnica. La introducción de nuevos métodos de cromatografía líquida acoplada a la MS/MS (LC-MS/MS) como ensayos de segundo nivel, para discriminar entre analitos isobáricos y entre diferentes patologías asociadas a un mismo marcador, permite disminuir el número de falsos positivo utilizando la misma muestra inicial.

En este sentido, La Marca y cols desarrollaron un método que permite monitorear y cuantificar los ácidos 3-hidroxi-propiónico y metilmalónico de forma independiente y diferencial de sus compuestos isobáricos: ácido láctico y ácido succínico respectivamente, mediante una corrida cromatográfica de cinco minutos a partir del mismo disco de sangre seca en papel de filtro utilizado para el resto de las acilcarnitinas y aminoácidos ${ }^{40}$. La aplicación de este método a muestras cuyo reanálisis inicial fue solicitado por un valor anormal de C3, permitió diferenciar completamente a las muestras realmente positivas así como discriminar entre una aciduria propiónica o metilmalónica. La tasa de falsos positivo se redujo de $22 \%$ a cero ${ }^{40}$.

Por otro lado, Shigematsu y cols han desarrollado un método de LC-MS/MS como prueba de segundo nivel para todas aquellas muestras con una concentración de C5 elevada. Esto permite eliminar la interferencia de algunos antibióticos en el diagnóstico de la acidemia isovalérica ${ }^{41}$.

La utilización de la LC-MS/MS como prueba 
de segundo nivel también ha posibilitado la separación y cuantificación de los aminoácidos isobáricos leucina, isoleucina, valina y alo-isoleucina, aumentando la especificidad del resultado del tamiz neonatal para la enfermedad de la orina con olor a jarabe de $\operatorname{arce}^{42}$.

Otro ejemplo en el que se ha aplicado este enfoque es en la detección de la tirosinemia tipo I. El programa de tamiz neonatal del estado norteamericano de Minnesota ha implementado un método de LC-MS/MS para determinar la concentración de succinilacetona cuando las concentraciones de tirosina en el tamiz inicial por el método de MS/MS tradicional se encuentran por encima del punto de corte establecido ${ }^{43}$. Aunque este acercamiento elimina de forma efectiva los resultados falsos positivos, aproximadamente $25 \%$ de los pacientes pueden no ser diagnosticados debido a que la concentración de tirosina en las muestras neonatales tomadas se encuentran por debajo de este valor ${ }^{44}$.

Existen diversos factores que afectan el perfil de metabolitos obtenido por MS/MS e influyen sobre el desempeño del tamiz. En este sentido, la razón de $\mathrm{C} 3 / \mathrm{C} 16$ resulta útil como marcador secundario para la detección de las acidurias propiónica y metilmalónica durante el período neonatal. Sin embargo, la rápida disminución de los niveles de acilcarnitinas de cadena corta en el tiempo en relación con las de cadena larga afecta su utilidad ${ }^{45}$. De forma general, tanto las concentraciones de carnitina libre como de las diferentes especies de acilcarnitinas varían significativamente a partir de la primera semana de nacido. En relación al sexo sólo se han reportado diferencias en relación a las especies C14, C16 y C18: $1^{46}$.

También se ha reportado que el aumento en el porcentaje de hematocrito de la muestra produce un incremento significativo en los niveles de numerosas especies de acilcarnitinas y de carnitina libre, así como en la mayoría de los aminoácidos. Además, a valores bajos de hematocrito existe una mayor influencia de la localización del disco dentro de la gota de sangre seca, siendo las concentraciones más elevadas en la periferia que en el centro ${ }^{47}$.

\section{Conclusiones.}

Numerosos centros de tamiz alrededor del mundo ya han expandido sus programas neona- tales al incluir un mayor número de enfermedades a detectar mediante la utilización de la MS/MS. Esta metodología ofrece importantes ventajas con respecto a los métodos tradicionales de tamiz, pues posibilita el seguimiento de diferentes biomarcadores de manera conjunta y la detección simultánea de un gran número de EIM a partir de una misma muestra. Además, la identificación de variaciones en la concentración de varios metabolitos relacionados con una misma patología ha posibilitado un incremento en la sensibilidad y especificidad del diagnóstico de diferentes EIM como la PKU y la MCAD ${ }^{4,48}$.

Por otro lado, aunque la MS/MS no permite distinguir entre algunos EIM detectados mediante el seguimiento de biomarcadores isómeros; la identificación de un posible enfermo es sólo el primer paso del tamiz neonatal. A este resultado sigue un proceso de confirmación que sí debe posibilitar esta discriminación. En este sentido, la utilización de métodos de LC-MS/MS como pruebas de segundo nivel ha permitido una disminución en el número de falsos positivo.

La aplicación de novedosas herramientas bioinformáticas y el incremento de las bases de datos también deben posibilitar la identificación de nuevos patrones de marcadores específicos para cada EIM.

\section{Referencias}

1. Campos Hernández D. Tamizaje neonatal por espectrometría de masas en tándem: actualización. Rev Panam Salud Publica 2010; 27: 309-18.

2. Lukacs Z, Santer R. Evaluation of electrospray-tandem mass spectrometry for the detection of phenylketonuria and other rare disorders. Mol Nutr Food Res 2006; 50: 443-50.

3. Schulze A, Lindner M, Kohlmüller D, Olgemöller K, Mayatepek E, Hoffmann GF. Expanded newborn screening for inborn errors of metabolism by electrospray ionization-tandem mass spectrometry: results, outcome, and implications. Pediatrics 2003; 11: 1399-406.

4. Ceglarek U, Müller P, Stach B, Bührdel P, Thiery J, Kiess $\mathrm{W}$. Validation of the phenylalanine/tyrosine ratio determined by tandem mass spectrometry: sensitive newborn screening for phenylketonuria. Clin Chem Lab Med 2002; 40: 693-7.

5. Zytkovicz TH, Fitzgerald EF, Marsden D, Larson CA, Shih VE, Johnson DM, et al. Tandem mass spectrometric 
analysis for amino, organic, and fatty acid disorders in newborn dried blood spots: a two-year summary from the New England Newborn Screening Program. Clin Chem 2001; 47: 1945-55.

6. Kasper DC, Ratschmann R, Metz TF, Mechtler TP, Möslinger D, Konstantopoulou V, et al. The national Austrian newborn screening program- eight years experience with mass spectrometry. Past, present, and future goals. Wien Klin Wochenschr 2010; 122: 607-13.

7. Oglesbee D, Sanders KA, Lacey JM, Magera MJ, Casetta B, Strauss KA, et al. Second-tier test for quantification of alloisoleucine and branched-chain amino acids in dried blood spots to improve newborn screening for maple syrup urine disease (MSUD). Clin Chem 2008; 54: 5429.

8. De Jesús VR, Chace DH, Lim TH, Mei JV, Hannon WH. Comparison of amino acids and acylcarnitines assay methods used in newborn screening assays by tandem mass spectrometry. Clin Chim Acta 2010; 411: 684-9.

9. Schutzman DL, Porat R, Salvador A, Janeczko M. Neonatal nutrition: a brief review. World J Pediatr 2008; 4 : 248-53.

10. Puckett RL, Lorey F, Rinaldo P, Lipson MH, Matern D, Sowa ME, et al. Maple syrup urine disease: further evidence that newborn screening may fail to identify variant forms. Mol Genet Metab 2010; 100: 136-42.

11. Oglesbee D, Sanders KA, Lacey JM, Magera MJ, Casetta B, Strauss KA, et al. Second-tier test for quantification of alloisoleucine and branched-chain amino acids in dried blood spots to improve newborn screening for maple syrup urine disease (MSUD). Clin Chem 2008; 54: 542-9.

12. Chace DH, Kalas T, Naylor EW. Use of tandem mass spectrometry for multianalyte screening on dried blood specimens from newborns. Clin Chem 2003; 49: 1797817.

13. Torres-Sepúlveda MR, Martínez-De Villarreal LE, Esmer C, González-Alanís R, Ruiz-Herrera C, Sánchez-Peña A, et al. Tamiz metabólico neonatal por espectrometría de masas en tándem: dos años de experiencia en Nuevo León, México. Salud Publica Mex 2008; 50: 200-6.

14. Allard P, Grenier A, Korson MS, Zytkovicz TH. Newborn screening for hepatorenal tyrosinaemia by tandem mass spectrometry: analysis of succinylacetone extracted from dried blood spots. Clin Biochem 2004; 37: 1010-5.

15. Chace DH, Lim T, Hansen CR, De Jesus VR, Hannon WH. Improved MS/MS analysis of succinylacetone extracted from dried blood spots when combined with amino acids and acylcarnitine butyl esters. Clin Chim Acta 2009; 407: 6-9.

16. Dhillon KS, Bhandal AS, Aznar CP, Lorey FW, Neogi P.
Improved tandem mass spectrometry (MS/MS) derivatized method for the detection of tyrosinemia type I, amino acids and acylcarnitine disorders using a single extraction process. Clin Chim Acta. 2011 Jan 7.

17. Matern D, Tortorelli S, Oglesbee D, Gavrilov D, Rinaldo P. Reduction of the false-positive rate in newborn screening by implementation of MS/MS-based second-tier tests: the Mayo Clinic experience (2004-2007). J Inherit Metab Dis 2007; 30: 585-92.

18. Rhead WJ. Newborn screening for medium-chain acylCoA dehydrogenase deficiency: a global perspective. J Inherit Metab Dis 2006; 29: 370-7.

19. Chace DH, Kalas TA, Naylor EW. Use of tandem mass spectrometry for multianalyte screening of dried blood specimens from newborns. Clin Chem 2003; 49: 1797817.

20. Van Hove JL, Zhang W, Kahler SG, Roe CR, Chen YT, Terada N, et al. Medium-chain acyl-CoA dehydrogenase (MCAD) deficiency: diagnosis by acylcarnitine analysis in blood. Am J Hum Genet 1993; 52: 958-66.

21. Okun JG, Kölker S, Schulze A, Kohlmüller D, Olgemöller $\mathrm{K}$, Lindner $\mathrm{M}$, et al. A method for quantitative acylcarnitine profiling in human skin fibroblasts using unlabelled palmitic acid: diagnosis of fatty acid oxidation disorders and differentiation between biochemical phenotypes of MCAD deficiency. Biochim Biophys Acta 2002; 1584: 91-8.

22. Koeberl DD, Young SP, Gregersen NS, Vockley J, Smith WE, Benjamin DK Jr, et al. Rare disorders of metabolism with elevated butyryl- and isobutyryl-carnitine detected by tandem mass spectrometry newborn screening. Pediatr Res 2003; 54: b219-23.

23. Chace DH, Diperna JC, Mitchell BL, Sgroi B, Hofman LF, Naylor EW. Electrospray tandem mass spectrometry for analysis of acylcarnitines in dried postmortem blood specimens collected at autopsy from infants with unexplained cause of death. Clin Chem 2001; 47: 1166-82.

24. Sim KG, Wiley V, Carpenter K, Wilcken B. Carnitine palmitoyltransferase I deficiency in neonate identified by dried blood spot free carnitine and acylcarnitine profile. J Inherit Metab Dis 2001; 24: 51-9.

25. Fingerhut R, Ensenauer R, Röschinger W, Arnecke R, Olgemöller B, Roscher AA. Stability of acylcarnitines and free carnitine in dried blood samples: implications for retrospective diagnosis of inborn errors of metabolism and neonatal screening for carnitine transporter deficiency. Anal Chem 2009; 81: 3571-5.

26. Fingerhut R, Röschinger W, Muntau AC, Dame T, Kreischer J, Arnecke R, et al. Hepatic carnitine palmitoyltransferase I deficiency: acylcarnitine profiles in blood spots are highly specific. Clin Chem 2001; 47: 1763-8. 
27. Wilcken B. Disorders of the carnitine cycle and detection by newborn screening. Ann Acad Med Singapore 2008; 37: 71-3.

28. Sigauke E, Rakheja D, Kitson K, Bennett MJ. Carnitine palmitoyltransferase II deficiency: a clinical, biochemical, and molecular review. Lab Invest 2003; 83: 1543-54.

29. Chace DH, Diperna JC, Kalas RA, Johnson RW, Naylor EW. Rapid Diagnosis of Methylmalonic and propionic acidemias: quantitative tandem mass spectrometric analysis of propionylcarnitine in filter-paper blood specimens obtained from newborns. Clin Chem 2001; 47: 2040-4.

30. Fletcher J. Disorders of vitamin B12 metabolism presenting through newborn screening. Ann Acad Med Singapore 2008; 37: 79-2.

31. Nennstiel-Ratzel U, Blankenstein O, Ceglarek U, Starke I, Stoppsack M, Rauterberg E, et al. National Screening Report 2004. [monografía en Internet]. Deutsche Gesellschaft für Neugeborenenscreening (DGNS); 2004. Disponible en: http://www.screening-dgns.de/PDF/ Screeningreport_2004_e.pdf [Consultado el 25 de agosto de 2009].

32. Lindner M, Ho S, Kölker S, Abdoh G, Hoffmann GF, Burgard P. Newborn screening for methylmalonic acidurias-optimization by statistical parameter combination. J Inherit Metab Dis 2008; 31: 379-85.

33. Cheng KH, Liu MY, Kao CH, Chen YJ, Hsiao KJ, Liu TT, et al. Newborn screening for methylmalonic aciduria by tandem mass spectrometry: 7 years' experience from two centers in Taiwan. J Chin Med Assoc 2010; 73: 314-8.

34. Wilcken B, Wiley V, Hammond J, Carpenter K. Screening newborns for inborn errors of metabolism by tandem mass spectrometry. N Engl J Med 2003; 348: 2304-12.

35. Baumgartner C, Baumgartner D. Biomarker discovery, disease classification, and similarity query processing on high-throughput MS/MS data of inborn errors of metabolism. J Biomol Screen 2006; 11: 90-9.

36. Chace DH, Kalas TA. A biochemical perspective on the use of tandem mass spectrometry for newborn screening and clinical testing. Clin Biochem 2005; 38: 296-309.

37. Wei CC, Lin WD, Tsai FJ, Wu JY, Peng CT, Tsai CH. Isovaleric acidemia diagnosed promptly by tandem mass spectrometry: report of one case. Acta Paediatr Taiwan 2004; 45: 236-8.

38. Van Calcar SC, Gleason LA, Lindh H, Hoffman G, Rhead W, Vockley G, et al. 2-methylbutyryl-CoA dehydrogenase deficiency in Hmong infants identified by expanded newborn screen. WMJ 2007; 106: 12-5.

39. Shigematsu Y, Hata I, Kikawa Y, Mayumi M, Tanaka Y, Sudo M, et al. Modifications in electrospray tandem mass spectrometry for a neonatal-screening pilot study in Japan. J Chromatogr B, Biomed Sci Apl 1999; 731: 97-103.

40. La Marca G, Malvagia S, Pasquini E, Innocenti M, Donati MA, Zammarchi E. Rapid 2nd-tier test for measurement of 3-OH-propionic and methylmalonic acids on dried blood spots: reducing the false-positive rate for propionylcarnitine during expanded newborn screening by liquid chromatography-tandem mass spectrometry. Clin Chem 2007; 53: 1364-9.

41. Shigematsu Y, Hata I, Tanaka Y. Stable-isotope dilution measurement of isovalerylglycine by tandem mass spectrometry in newborn screening for isovaleric academia. Clin Chim Acta 2007; 386: 82-6.

42. Frazier DM. Tyrosine results in MS/MS newborn screening: the highs and lows. Newborn Screening and Genetic Testing Symposium. [monografía en Internet] Minneapolis; 2007. Disponible en: http://www.aphl.org/ profdev/conferences/proceedings/Documents/2007_ NBS_and_Genetic_Testing_Symposium/Tyrosine_Results_in_NBS.pdf [Consultado el 22 de octubre de 2009].

43. Magera MJ, Gunawardena ND, Hahn SH, Tortorelli S, Mitchell GA, Goodman SI, et al. Quantitative determination of succinylacetone in dried blood spots for newborn screening of tyrosinaemia type I. Mol Genet Metab 2006; 88: 16-21.

44. Matern D, Tortorelli S, Oglesbee D, Gavrilov D, Rinaldo P. Reduction of the false-positive rate in newborn screening by implementation of MS/MS-based second-tier tests: the Mayo Clinic experience (2004-2007). J Inherit Metab Dis 2007; 30: 585-92.

45. Fingerhut R, Ensenauer R, Röschinger W, Arnecke R, Olgemöller B, Roscher AA. Stability of acylcarnitines and free carnitine in dried blood samples: implications for retrospective diagnosis of inborn errors of metabolism and neonatal screening for carnitine transporter deficiency. Anal Chem 2009; 81: 3571-5.

46. Cavedon CT, Bourdoux P, Mertens K, Van Thi HV, Herremans N, De Laet C. Age-related variations in acylcarnitine and free carnitine concentrations measured by tandem mass spectrometry. Clin Chem 2005; 51: 745-52.

47. Holub M, Tuschl K, Ratschmann R, Strnadová KA, Mühl A, Heinze G, et al. Influence of hematocrit and localisation of punch in dried blood spots on levels of amino acids and acylcarnitines measured by tandem mass spectrometry. Clin Chim Acta 2006; 373: 27-31.

48. Tran K, Banerjee S, Li H, Noorani HZ, Mensinkai S, Dooley K. Clinical efficacy and cost-effectiveness of newborn screening for medium chain acyl-CoA dehydrogenase deficiency using tandem mass spectrometry. Clin Biochem 2007; 40: 235-41. 